\title{
Are Atrial High-rate Episodes a Kind of Sympathetic Overactivity in Patient with Permanent Pacemaker?
}

\section{هi Ömer Çağlar Yılmaz}

Private Cardiology Clinic, Ankara, Turkey

\begin{abstract}
Objectives: Atrial high-rate episodes (AHREs) are important cardiac conditions. The purpose of the trial was to assess sympatho-vagal interaction as evaluated by the heart rate variability (HRV) of holter recordings in patients with AHRE.

Materials and Methods: All subjects with cardiac devices including dual chamber permanent pacemakers, attending the outpatient pacemaker control units were included. Transthoracic echocardiography and a rest electrocardiogram, and 24-hour Holter monitoring were performed to all patients.

Results: One-hundred and fifty subjects were included in this trial. There were 44 patients (29.3\%) with AHRE and 106 subjects $(70.7 \%)$ without AHRE in all population. Standard deviation of all N-N intervals for a selected time period $(102.1 \pm 30.5$ vs $124.0 \pm 50.2 ; \mathrm{p}=0.008)$ and normalized power in high-frequency band $(9.4 \pm 6.6,12.7 \pm 7.1 ; \mathrm{p}=0.006)$ were significantly decreased; however, normalized power in low-frequency band $(28.5 \pm 12.5,23.8 \pm 11.6 ; \mathrm{p}=0.006)$ and LF/HF ratio $(4.71 \pm 3.60,2.63 \pm 1.65 ; \mathrm{p}<0.001)$ were significantly increased in AHRE patients.
\end{abstract}

Conclusion: There was a sympathetic overactivity in patients with AHRE. Further studies are needed to demonstrate the role of HRV parameters in AHRE patients.

Keywords: Atrial high-rate episodes (AHRE), HRV, autonomic nervous system

Address for Correspondence: Ömer Çağlar Yılmaz, Private Cardiology Clinic, Ankara, Turkey

e-mail: drocaglaryilmaz@hotmail.com ORCID: orcid.org/0000-0001-5245-5216

Received: 30.01.2021 Accepted: 15.02.2021

Cite this article as: Çağlar Ö. Are Atrial High-rate Episodes a Kind of Sympathetic Overactivity in Patient with Permanent

Pacemaker?. EJCM 2021;9(1):49-52.

DOI: $10.32596 /$ ejcm.galenos.2021-01-07

${ }^{\circ}$ Copyright 2021 by Heart and Health Foundation of Turkey (TÜSAV) / E Journal of Cardiovascular Medicine published by Galenos Publishing House. 


\section{Introduction}

Atrial high-rate episodes (AHREs), known as subclinical arrhythmias, are notable cardiac conditions. Atrial high-rate episode can be described as an episode of faster heart rate, generally higher than 180 beats/ minute lasting at least 5 minutes according to the current guidelines ${ }^{(1)}$. AHRE can be recognized by documentation type from atrial fibrillation (AF). AF is detected on an electrocardiogram or Holter monitoring; however, AHREs are merely recorded on a cardiac implantable electronic devices (CIED) read-out ${ }^{(1)}$. The rate of AHRE can reach $50 \%$.

Heart rate variability (HRV) has some parameters which represent sympato-vagal interaction of the sinoatrial node (SAN). It is also related to autonomic modulation of SAN. It has been studied to identify the role of autonomic nervous system (ANS) activity in different cardiovascular conditions $^{(3)}$.

The purpose of the trial was to assess sympatho-vagal interaction as evaluated by the HRV of Holter recordings in subjects with AHRE.

\section{Materials and Methods}

Patients with CIEDs including dual chamber permanent pacemakers and attending the outpatient pacemaker control unit of three Cardiovascular Centers from March 2010 to February 2019 were enrolled to the study. Patients with heart failure, chronic obstructive pulmonary disease, acute coronary syndrome, previous myocardial infarction, AF, those with decreased ejection fraction $(<40 \%)$, and those with implantable defibrillator or cardiac resynchronization therapy device were excluded due to heart failure that could impair HRV parameters. We also excluded fulltime pacemaker dependent patients and single-chamber VVI devices. Atrial sensitivity was programmed to $0.5 \mathrm{mV}$ with bipolar sensing.

Clinical assessment, laboratory test, echocardiography and 12 lead electrocardiogram before the Holter recording were done to all subjects.

Ömer Çağlar Yılmaz. AHRE and Sympatetic Overactivity
Ethics committee approval was obtained from University of Health Sciences Turkey, Ankara Keçiören Training and Research Hospital Clinical Researches Ethics Committee (decision no: KAEK2013-18, date: 12.04. 2013).

\section{Holter Monitoring}

CONTEC Holter system was utilized to handle the Holter analysis.

All 24-hour recordings were utilized to assess HRV indexes. Time-domain and spectral results of HRV were calculated using the guideline of $\mathrm{ESC}^{(4)}$.

The standard parameters [standard deviation of all NN intervals for a selected time period (SDNN), square root of the mean of the sum of the squares of differences between adjacent RR intervals (RMSSD) and the proportion of differences in successive $\mathrm{NN}$ intervals greater than 50 $\mathrm{ms}$ (pNN50)] and spectral analysis [high-frequency (HF) component $(0.15-0.40 \mathrm{~Hz})$, low-frequency (LF) component (0.04-0.15 Hz)] were used for HRV analysis.

The normalized high-frequency power $(\mathrm{HFnu})=100 x h i g h$-frequency power/total power, normalized low-frequency power $(\mathrm{LFnu})=100 x \mathrm{xlow}-$ frequency power/total power, and low/high-frequency power ratio low-frequency power/high-frequency power (LF/HF ratio) were calculated. LF/HF ratio reflects sympatovagal balance (higher 2.5 reflects sympatetic, lower 2.5 reflects parasympaptetic overactivity) ${ }^{(4)}$.

\section{Transthoracic Echocardiography}

All subjects underwent transthoracic echocardiography by using a Philips affinity 50 echocardiography machine.

\section{Statistical Analysis}

The Pearson chi-square test, ANOVA and MannWhitney U-test were used to compare variables. For post-hoc analysis, the Tukey test was utilized. SPSS 15.0 software was used.

\section{Results}

One-hundred and fifty subjects were included in this trial. Patient characteristics and echocardiography results 
are demonstrated in Table 1 . There were 44 patients (29.3\%) with AHRE and 106 subjects (70.7\%) without AHRE in all population. No significant difference was present between two groups in terms of age and gender ratio. Patients with AHRE had increased left atrial diameter and left atrial area, whereas cardiac diastolic functional parameters assessed by mitral valve Continuous Wave Doppler indexes were similar. It was demonstrated in Table 1.

HRV parameters of all subjects were shown in Table 2. SDNN (102.1 \pm 30.5 vs $124.0 \pm 50.2 ; \mathrm{p}=0.008)$ and HFnu (9.4 $\pm 6.6,12.7 \pm 7.1 ; p=0.006)$ were significantly lower. LFnu $(28.5 \pm 12.5,23.8 \pm 11.6 ; \mathrm{p}=0.006)$ and $\mathrm{LF} / \mathrm{HF}$ ratio $(4.71 \pm 3.60,2.63 \pm 1.65 ; \mathrm{p}<0.001)$ were significantly higher in AHRE patients (Figure 1). The alterations in LF nu and LF/HF ratio showed a higher sympathetic tone and disparity in autonomic activity in AHRE subjects.

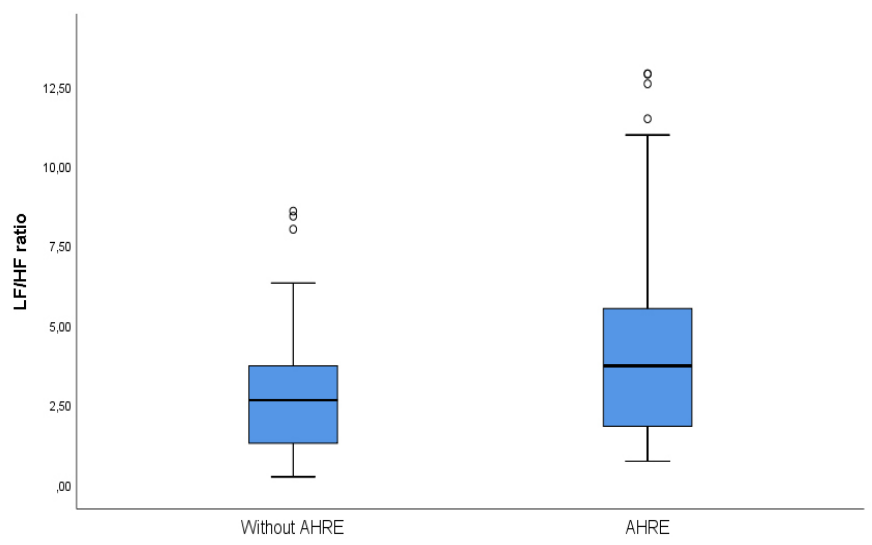

Figure 1. LF/HF ratio of Subjects with AHRE and without AHRE LF: Low frequency, HF: High frequency, AHRE: Atrial high rate episodes

Table 1. Baseline characteristics of the patients with AHRE and without AHRE

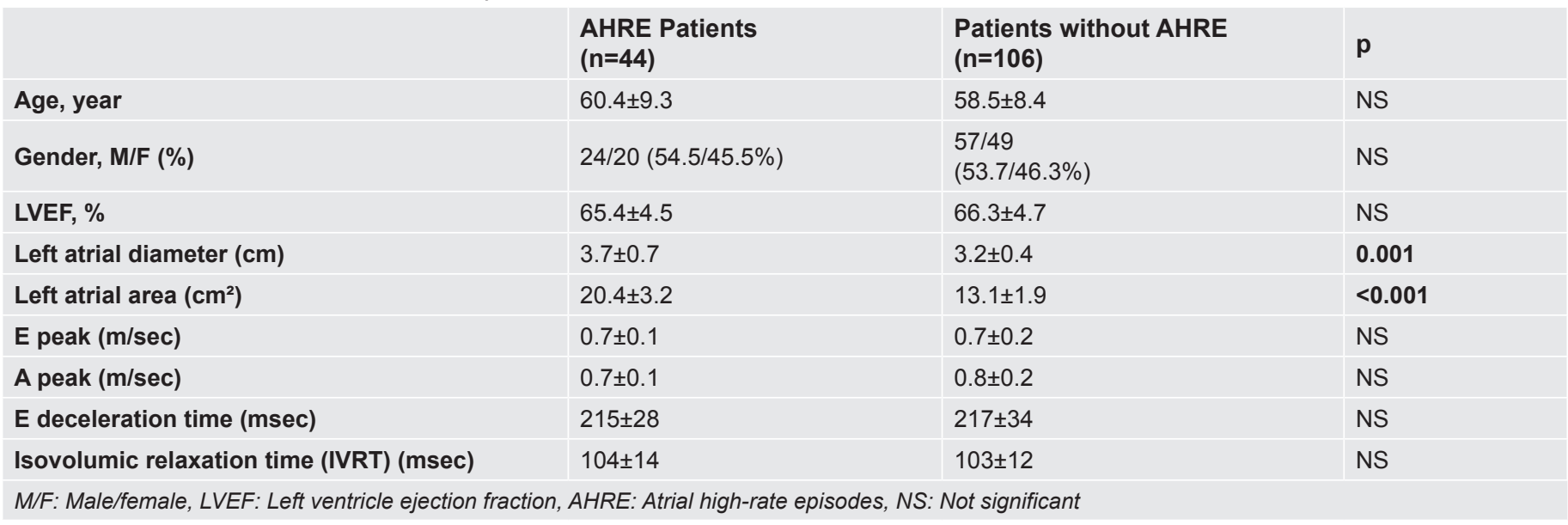

Table 2. HRV parameters in subjects with AHRE and without AHRE

\begin{tabular}{|c|c|c|c|}
\hline & AHRE subjects & Subjects without AHRE & $\mathbf{p}$ \\
\hline Heart rate & $81.2 \pm 17.5$ & $73.8 \pm 14.2$ & $<0.001$ \\
\hline SDNN (ms) & $102.1 \pm 30.5$ & $124.0 \pm 50.2$ & $<0.001$ \\
\hline PNN50 (\%) & $11,7 \pm 1,5$ & $10 \pm 1,4$ & NS \\
\hline 24-hour LFnu & $28.5 \pm 12.5$ & $23.8 \pm 11.6$ & 0.02 \\
\hline
\end{tabular}


Any correlation could not be found between any HRV parameters and demographic features of the subjects.

\section{Discussion}

In the study, we showed that patients with AHRE had more impaired HRV parameters reflecting sympathetic overactivity compared to those without AHRE. To the best of our knowledge, this is the first study examining HRV parameters in patients with AHRE in preserved LV systolic function.

AHREs are not a rare condition in subjects having intra-cardiac devices without AF after development in cardiovascular follow up. Atrial high-rate episodes can be observed highly in next times. Atrial high-rate episodes are usually thought of similar to clinical paroxysmal AF. It could be given support by former trials showing that AHREs have an increased relation to clinical $\mathrm{AF}^{(5)}$.

HRV parameters reflect the autonomic activity on the heart. It is related to alterations in autonomic balance. In the study, the deterioration of both various parameters of HRV in AHRE patients was shown. It has been mentioned that disrupted sympatho-vagal interaction for the good of sympathetic system can be caused by heart repolarization abnormalities and tachyarrythmias ${ }^{(6)}$.

Several risk factors such as inflammation, autonomic changes, high pressure or volume burden can play roles as potential agents to cause a favorite substrate for AHRE creation ${ }^{(7)}$. Various trials have investigated that different promoting agents to atrial arrhythmias look like autonomic layout. Parasympathetic activation is demonstrated to decrease the atrial effective refractory period and tendency to atrial arrhythmias ${ }^{(8)}$. In some cardiac disorders, sympathetic activity plays a crucial role in atrial arrhythmia initiation ${ }^{(9)}$. In this study, higher sympathetic tone in patients with AHRE was also found.

\section{Conclusion}

There was a sympathetic overactivity in patients with AHRE in the current study. Further larger trials are needed to demonstrate the role of HRV parameters in AHRE patients.

\section{Ethics}

Ethics Committee Approval: This study was approved by University of Health Sciences Turkey, Ankara Keçiören Training and Research Hospital Clinical Researches Ethics Committee (decision no: KAEK201318, date: 12.04. 2013).

Informed Consent: Informed consent was obtained from all participants.

Peer-review: Externally peer-reviewed.

Financial Disclosure: There is no funding source

\section{References}

1. Kirchhof P, Benussi S, Kotecha D, et al. 2016 ESC Guidelines for the management of atrial fibrillation developed in collaboration with EACTS. Eur Heart J 2016;37:2893-962.

2. Chen-Scarabelli C, Scarabelli TM, Ellenbogen KA, Halperin JL. Devicedetected atrial fibrillation: what to do with asymptomatic patients? J Am Coll Cardiol 2015;65:281-94.

3. Pumprla J, Howorka K, Groves D, Chester M, Nolan J. Functional assessment of heart rate variability: physiological basis and practical applications. Int J Cardiol 2002;84:1-14.

4. Task Force of European Society of Cardiology and the North American Society of Pacing and Electrophysiology on Heart Rate Variability. Standards of measurement, physiological interpretation, and clinical use. European Heart Journal 1996;17:354-81.

5. Pollak WM, Simmons JD, Interian A Jr, et al. Clinical utility of intraatrial pacemaker stored electrograms to diagnose atrial fibrillation and flutter. Pacing Clin Electrophysiol 2001;24:424-9.

6. Valensi PE, Johnson NB, Maison-Blanche P, Extramania F, Motte G Coumel P. Influence of cardiac autonomic neuropathy on heart rate dependence of ventricular repolarization in diabetic patients. Diabetes Care 2002;25:918-23

7. Goldberger JJ, Arora R, Green D, et al. Evaluating the Atrial Myopathy Underlying Atrial Fibrillation: Identifying the Arrhythmogenic and Thrombogenic Substrate. Circulation 2015;132:278-91.

8. Hirose M, Leatmanoratn Z, Laurita KR, Carlson MD. Partial vagal denervation increases vulnerability to vagally induced atrial fibrillation. J Cardiovasc Electrophysiol 2002;13:1272-9.

9. Lu Z, Scherlag BJ, Lin J, et al. Atrial fibrillation begets atrial fibrillation: autonomic mechanism for atrial electrical remodeling induced by shortterm rapid atrial pacing. Circ Arrhythm Electrophysiol 2008;1:184-92. 